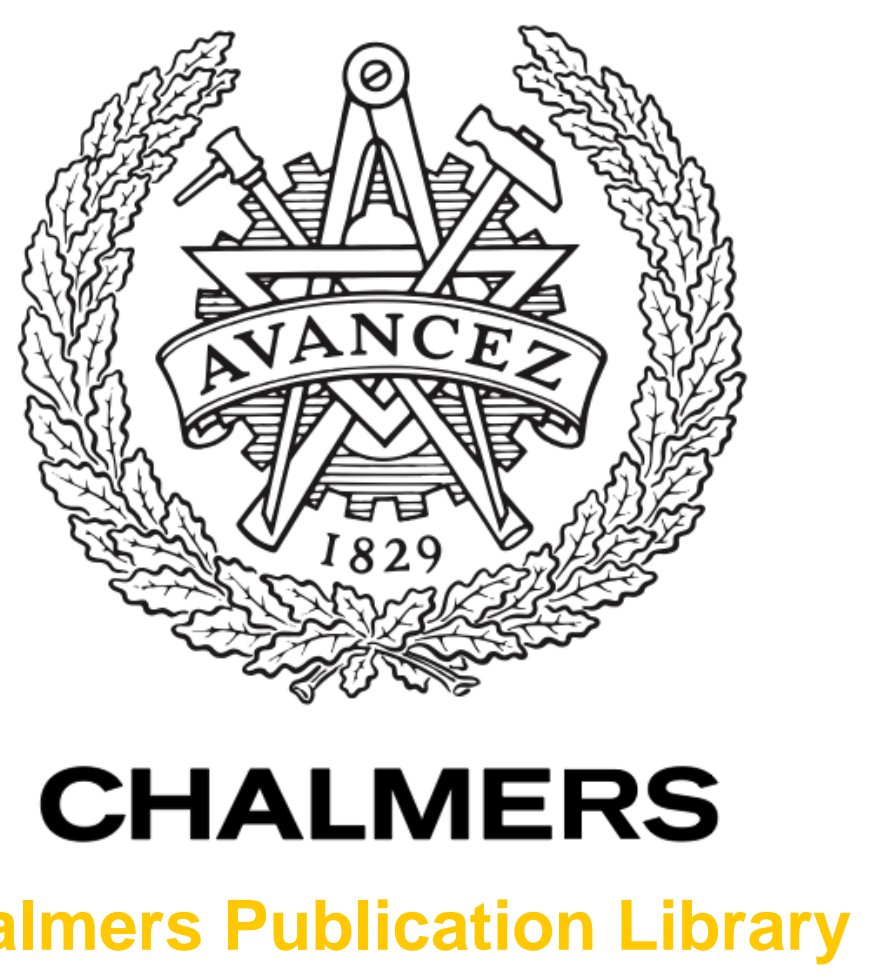

Chalmers Publication Library

\title{
Neuronal cell growth on polymeric scaffolds studied by CARS microscopy
}

This document has been downloaded from Chalmers Publication Library (CPL). It is the author's version of a work that was accepted for publication in:

Proceedings of SPIE - Multiphoton Microscopy in the Biomedical Sciences XII, San Francisco, CA, 22-24 January 2012 (ISSN: 1605-7422)

Citation for the published paper:

Enejder, A. ; Fink, H. ; Kuhn, H. (2012) "Neuronal cell growth on polymeric scaffolds studied by CARS microscopy". Proceedings of SPIE - Multiphoton Microscopy in the Biomedical Sciences XII, San Francisco, CA, 22-24 January 2012, vol. 8226

http://dx.doi.org/10.1117/12.910134

Downloaded from: http://publications.lib.chalmers.se/publication/157058

Notice: Changes introduced as a result of publishing processes such as copy-editing and formatting may not be reflected in this document. For a definitive version of this work, please refer to the published source. Please note that access to the published version might require a subscription.

Chalmers Publication Library (CPL) offers the possibility of retrieving research publications produced at Chalmers University of Technology. It covers all types of publications: articles, dissertations, licentiate theses, masters theses, conference papers, reports etc. Since 2006 it is the official tool for Chalmers official publication statistics. To ensure that Chalmers research results are disseminated as widely as possible, an Open Access Policy has been adopted.

The CPL service is administrated and maintained by Chalmers Library. 


\title{
Neuronal cell growth on polymeric scaffolds studied by CARS microscopy
}

\author{
Annika Enejder ${ }^{1}$, Helen Fink ${ }^{1}$, and Hans-Georg Kuhn ${ }^{2}$ \\ ${ }^{1}$ Chalmers University of Technology, Department of Chemical and Biological Engineering, Molecular Microscopy, \\ Göteborg, Sweden \\ ${ }^{2}$ University of Gothenburg, Institute of Neuroscience and Physiology, Göteborg, Sweden
}

\begin{abstract}
For studies of neuronal cell integration and neurite outgrowth in polymeric scaffold materials as a future alternative for the treatment of damages in the neuronal system, we have developed a protocol employing CARS microscopy for imaging of neuronal networks. The benefits of CARS microscopy come here to their best use; (i) the overall three-dimensional (3D) arrangement of multiple cells and their neurites can be visualized without the need for chemical preparations or physical sectioning, potentially affecting the architecture of the soft, fragile scaffolds and (ii) details on the interaction between single cells and scaffold fibrils can be investigated by close-up images at sub-micron resolution. The establishment of biologically more relevant 3D neuronal networks in a soft hydrogel composed of native Extra Cellular Matrix (ECM) components was compared with conventional two-dimensional networks grown on a stiff substrate. Images of cells in the hydrogel scaffold reveal significantly different networking characteristics compared to the $2 \mathrm{D}$ networks, raising the question whether the functionality of neurons grown as layers in conventional cultivation dishes represents that of neurons in the central and peripheral nervous systems.
\end{abstract}

\section{INTRODUCTION}

Major efforts are presently made to develop polymeric scaffold materials for guided integration, protective support and signal stimulation of neurons in order to treat damages in the central and peripheral nervous systems causing e.g. vision-, hearing- and motor impairments. Development of ideal scaffolds for nerve regeneration requires fundamental understanding for the impact of the biochemistry and architecture of the substrate on cell adhesion, proliferation and neurite extension. This requires insights into the mechanisms behind cell-substrate interactions at subcellular level, traditionally acquired by studies of two-dimensional (2D) cell cultures on the material. Although 2D culture systems provide useful information about many biological processes, there is an increasing awareness that the polar organization of cell-cell and cellscaffold contacts forms a synthetic cell line with altered metabolism, gene expression and morphology [1]. For neurons the consequence is particular adverse since their core functionality signal transmission - turns abnormal as a result of the $2 \mathrm{D}$ restriction in neurite outgrowth characterized by incomplete synapse formations [2] and distorted electrophysiological properties $[3,4]$. Thus, in order to get a more realistic picture of the interaction mechanisms between neurons/neurites and polymeric components in scaffolds of different architectures and/or macromolecular compositions, cells are preferably grown and studied as three-dimensional (3D) neural networks.

Multiphoton Microscopy in the Biomedical Sciences XII, edited by Ammasi Periasamy, Karsten König, Peter T. C. So, Proc. of SPIE Vol. 8226, 82261W · @ 2012 SPIE · CCC code: 1605-7422/12/\$18 · doi: 10.1117/12.910134 
However, these soft, fragile and optically thick samples are challenging to investigate. The scaffolds typically consist of hydrogels/networks of natural or synthetic hydrophilic polymers [5], held together by fairly weak intermolecular crosslinks and co-arrangements with water molecules. Cells adhere to the scaffold components through highly dynamic ligand-receptor complexes [6] characterized by rupture forces per surface area in the $\mathrm{nN} / \mu \mathrm{m}^{2}$ regime [7], making the interactions quite sensitive to properties in the immediate surroundings. There is a considerable risk that both the architecture of the scaffold and the cell adhesions to the material are affected by the methods used for characterization, many of which involve chemical treatments, dehydration, temperature variations and mechanical stress. Visual inspection of the samples by conventional bright-field, polarization, phase contrast or differential interference contrast microscopy belongs to the least harmful characterization techniques, however, they provide limited lateral resolution $(>1 \mu \mathrm{m})$ and no 3D information, making it impossible to resolve deep-laying cells and neurites. Most other microscopy techniques necessitate contrast enhancement and harsh sample preparation, making it questionable to which extent the architecture of the scaffold and associated cell adhesions are being conserved and studied. Electron microscopy requires the samples to be dehydrated and contrast is enhanced by metal coating [8], most likely modifying the structure of the wet scaffold materials and excluding the possibility to follow dynamic adhesion formation mechanisms in time and space. In addition, it merely provides access to the most superficial layer, requiring physical sectioning in order to examine the matrix interior, which in turn could introduce compression artifacts to the architecture. Atomic Force Microscopy (AFM) has been used with minor success as the tip sticks to the wet scaffold materials [9]. Fluorescence microscopy requires the addition of a fluorescent dye to the sample, frequently resulting in an inhomogeneous distribution of the fluorophore in thicker scaffolds creating an aberrant picture of the distribution of cells and scaffold components. Some fluorophores have even been reported to disrupt the fiber structure of biopolymer scaffolds [10] and the limited photo-stability of most dyes excludes studies of time-dependent phenomena. These limitations of current imaging techniques make the development of alternative approaches for non-destructive, label-free 3D imaging of soft and millimeter-sized samples of highest interest for the tissue engineering community. In this work, we show the strength of CARS microscopy for label-free, minimally invasive imaging of the 3D arrangement of neuronal networks in a hydrogel composed of native ECM components. Cell morphology and neurite outgrowth were studied and compared with corresponding parameters observed for conventional 2D networks grown on poly-lysin coated planar substrates. Detailed visual information on the arrangements of cell bodies and neurites could be obtained by probing the $\mathrm{CH}$-stretch vibration characteristic of membrane lipids and protein filaments. Significantly different cell morphology and neurite growth characteristics were observed depending on whether the cells were grown in $2 \mathrm{D}$ or $3 \mathrm{D}$ environments.

\section{MATERIALS AND METHODS}

\subsection{The microscope setup}

The non-linear microscope used for CARS microscopy is composed of an inverted Nikon Eclipse TE-2000 microscope equipped with a Nikon C1 scanning head and a pico-second pulsed laser system. The laser source consists of a Nd:Vanadate pump laser $(10 \mathrm{~W}, 7 \mathrm{ps}, 76 \mathrm{MHz}$, Picotrain, HighQ Lasers $\mathrm{GmbH}$, Hohenems, Austria) and a ring-cavity optical parametric oscillator 
(Emerald OPO, APE GmbH, Berlin, Germany). Two beams are couple out from the pump laser; at $1064 \mathrm{~nm}$ directly aligned into the microscope as well as at $532 \mathrm{~nm}$ seeding the OPO. The OPO has in turn a variable output in the wavelength range $690-990 \mathrm{~nm}$. Spatial overlap between the $1064 \mathrm{~nm}$ and the OPO beam is achieved by aligning them into a common optical path using dichroic mirrors. A delay line for the OPO beam is employed for temporal overlap. The beams are directed into the confocal microscope through a mirror scanning unit (Nikon C1) and focused on the sample by an objective (Nikon Plan Fluor 40x/1.30 oil or TIRF 100x/1.45 oil). Signals are detected in forward direction using a sensitive photomultiplier tube, PMT (PMC-100-1, Hamamatsu, Japan) connected to a time-correlated single-photon counting unit (SPCM-830, Becker\&Hickl, Berlin, Germany). Bandpass filters are used to isolate the CARS signals and suppress background light. In the present study the symmetric $\mathrm{CH}_{2}$ vibration at $2845 \mathrm{~cm}^{-1}$ was probed by the combination of the fundamental $(1064 \mathrm{~nm})$ of the Nd:Vanadate laser (Stokes) and the output of the OPO tuned to $817 \mathrm{~nm}$ (pump/probe beam).

\subsection{Sample preparation and cell seeding}

For growth of conventional 2D neural networks on planar substrates, petri dishes (Willco Wells, the Netherlands) were pre-treated with $5 \mu \mathrm{g} / \mathrm{cm}^{2}$ poly-D-lysin (Sigma Aldrich, Germany) for 5 $\mathrm{min}$. The solution was discarded and the surfaces air dried for at least $1 \mathrm{~h}$. For the preparation of 3D neural networks, petri dishes were covered with a $300 \mu \mathrm{m}$ thick layer of Matrigel (VWR International, Sweden), a solubilized basement membrane preparation extracted from the Engelbreth-Holm-Swarm sarcoma. It contains extracellular matrix proteins, growth factors, collagenases and other components found in the native ECM. The Matrigel was thawed overnight on ice and was kept on ice until polymerization. The gel was mixed with cell culture medium 1:3 and transferred to the dishes. Thereafter, the dishes were placed in an incubator at $37^{\circ} \mathrm{C}$ for 30 min to allow polymerization of the gel.

1.

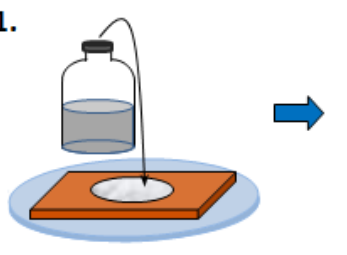

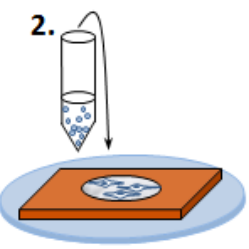

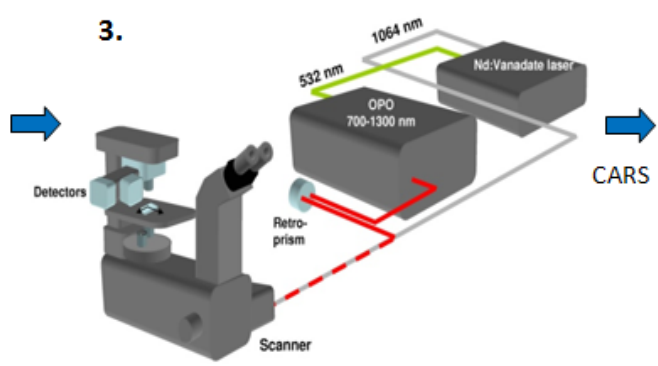

4.

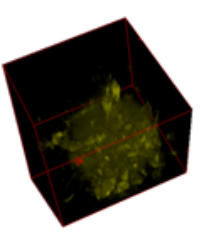

Figure 1. A schematic of the experimental outline: 1. Matrigel preparation, 2. cell seeding, 3. CARS microscopy and 4. image analysis. The CARS microscope shown in 3. consists of a pump laser, an optical parametric oscillator (OPO), and an inverted research microscope. The CARS signal is detected in the forward direction by a PMT coupled to a time-correlated single-photon counting unit.

Primary rat neurons (Gibco, Life Technologies Europe, the Netherlands) were thawed and seeded in Neurobasal medium (Gibco, Life Technologies Europe, the Netherlands) supplemented with $2 \%$ B27 supplement and $0.5 \mathrm{mM}$ glutamine at a density of $1 \times 10^{5}$ cells $/ \mathrm{cm}^{2}$ on both the $2 \mathrm{D}$ substrate and the Matrigel scaffold. Samples were incubated at standard physical conditions at $37^{\circ} \mathrm{C}$ in a humidified atmosphere of $5 \% \mathrm{CO}_{2}$ in air. Medium was changed every third day. For 
fixation, samples were rinsed twice with phosphate buffered saline (PBS), fixed with ice-cold methanol/aceton at a ratio of 1:1 for $10 \mathrm{~min}$. The fixation solution was discarded and the samples were rinsed twice with PBS. All samples were kept in PBS until measurements.

\section{RESULTS AND DISCUSSION}

\subsection{CARS spectrum of primary rat neurons}

In contrast to other cell categories, neurons contain minimal amounts of neutral lipids, such as triacylglycerols, stored as lipid droplets, leaving membrane lipids as their primary lipid constituent (77-86\%) [11]. The lack of dense lipid stores in the cytoplasm of neurons, which for other cell types has been proven to be an important contribution to the image contrast in CARS microscopy in the $\mathrm{CH}$-vibrational region [12] and the fact that they are embedded in a matrix consisting of a dense gel of proteins and water, motivates a more thorough analysis of the contrast mechanisms in the CARS images of neurons in Matrigel. Hence, a spectral series of CARS images over the high-frequency $\mathrm{CH}$-vibration range was collected (Fig. 2) to determine the resonant contributions.
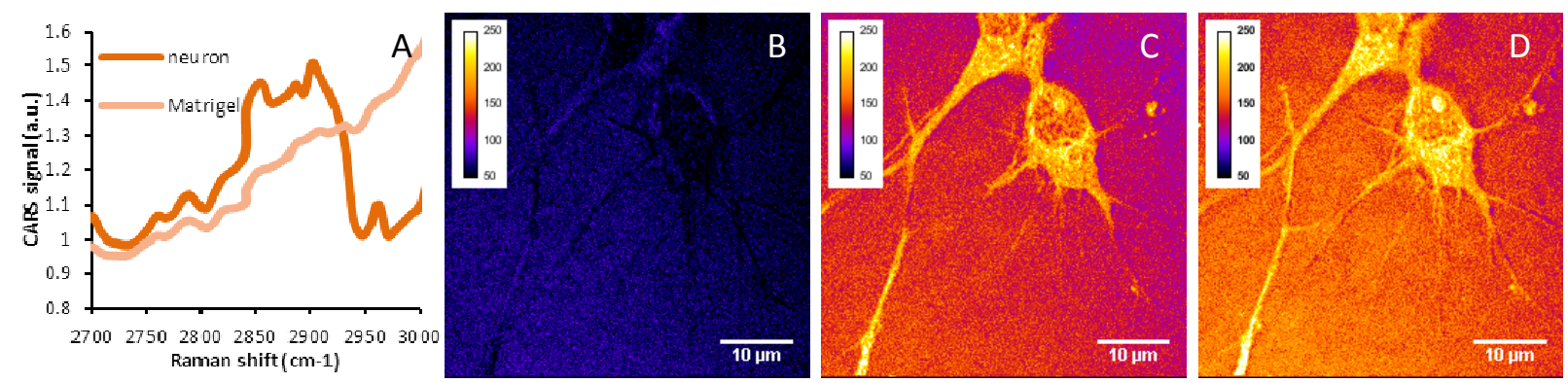

Figure 2. CARS spectra (A) evaluated from a series of CARS images on primary neurons in a Matrigel scaffold, collected while tuning vibrations in the $\mathrm{CH}$ vibrational region $\left(2700-3000 \mathrm{~cm}^{-1}\right)$. Corresponding CARS images at (B) $\sim 2700 \mathrm{~cm}^{-1}$ (non-resonant background) as well as at the (C) symmetric $\left(2845 \mathrm{~cm}^{-1}\right)$ and (D) asymmetric $\left(2880 \mathrm{~cm}^{-1}\right)$ $\mathrm{CH}_{2}$ vibrations are shown for comparison. Cell bodies and larger neurites show best contrast relative the Matrigel at $2845 \mathrm{~cm}^{-1}$, while the smaller neurites in the neurite growth cones are easier to distinguish at $2880 \mathrm{~cm}^{-1}$.

The CARS spectrum reveals that the neurons generate a broad resonant signal in the vibrational range 2840-2900 $\mathrm{cm}^{-1}$, including the symmetric and asymmetric $\mathrm{CH}_{2}$ vibrations at $2845 \mathrm{~cm}^{-1}$ and $2880 \mathrm{~cm}^{-1}$, characteristic for lipids due to their long acyl-chains. It is a factor of $\sim 1.4$ stronger than the non-resonant background, evaluated at $2675-2725 \mathrm{~cm}^{-1}$, which provides sufficient molecular contrast for imaging; compare Figs. 2B (non-resonant background) and 2C (CARS image at the $2845 \mathrm{~cm}^{-1}$ resonance). Interestingly, in contrast to CARS spectra collected on other cell categories with a lipid pool primarily consisting of triacylglycerols [12], the most prominent vibration occurs at $\sim 2880 \mathrm{~cm}^{-1}$ rather than at $2845 \mathrm{~cm}^{-1}$. This is characteristic for the most prominent lipids found in neurons and brain tissue (containing minimal amounts of triacylglycerols) such as phosphatidylcholine, sphingomyelin, gangliosides, and cholesterol [13]. This is further confirmed by a distinct vibration observed at $\sim 2960 \mathrm{~cm}^{-1}$, characteristic for the asymmetric $\mathrm{CH}_{3}$ vibration of the choline head group in phosphatidylcholine [14]. The CARS spectrum of the neuron is in Fig. 2A compared with that of the Matrigel, in order to evaluate best image contrast. The CARS spectrum of Matrigel exhibits a spectral shape which can be attributed 
to the low-frequency flank of the $\mathrm{OH}$ vibration [15] due to the high water content. From the spectrum we conclude that best image contrast for neurons in a background of Matrigel seems to be at $\sim 2850 \mathrm{~cm}^{-1}$ despite the stronger CARS signal at $\sim 2880 \mathrm{~cm}^{-1}$, see image in Fig. $2 \mathrm{C}$. However, smaller neurits with less lipid content (15\% dry weight relative to $35 \%$ in the soma [11]) may become easier to distinguish at $2880 \mathrm{~cm}^{-1}$, benefiting from the stronger CARS signal, see Fig. 2D.

\subsection{Morphology of neurons on 2D vs. 3D surface}

Neurons are clearly visible on both 2D control surfaces and Matrigel by probing the CH-vibration at $2845 \mathrm{~cm}^{-1}$, see Fig. 3. This molecular contrast together with the optical sectioning capabilities of CARS microscopy, give valuable three-dimensional information, from which morphological differences can be determined. The arrangement and morphology of neurons grown on a 2D glass surface differ considerably from that of cells on a 3D gel, as illustrated in the volume renderings in Figs. 3A and 3B. Cells grown on the surface of a petri dish readily adhere and develop neurites already after one day, though they display a synthetic, flat, bipolar morphology with larger, discrete contact areas merely on the ventral side of the cells toward the supporting surface. The interaction with other cells and neurite outgrowth is limited to the plane, thus, cells are less stimulated and develop few $(\sim 2)$ neurites per cell.
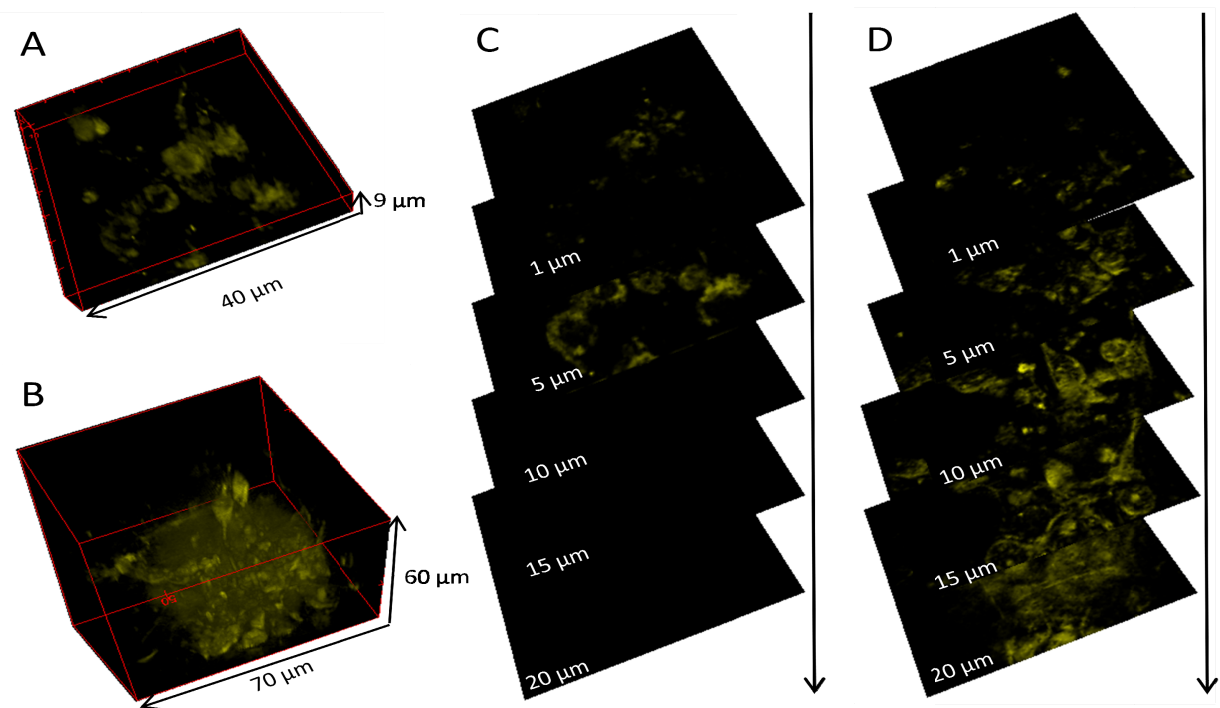

Figure 3. CARS microscopy images of cells grown on glass and Matrigel. 3D images reconstructed from z-stacks of CARS microscopy images (A and B). Images C and D show sections taken at different depth illustrating the different morphological characteristics of cells grown on a 2D surface (C) and on 3D surface (D).

The development of neurites for cells grown on Matrigel takes in general somewhat longer time, 2-4 days. However, the cells show a native phenotype and grow like a three-dimensional cluster with an extensive network of neurites ( $>4$ per cell) connecting cells located in different planes. For clarity, the different image planes of the volume rendering are shown in Figs. $3 \mathrm{C}$ and $\mathrm{D}$ at corresponding depths. While cells in the petri dish sample merely show up at depths 1 and $5 \mu \mathrm{m}$, complete integration into the full depth of the Matrigel is observed. The average thickness of the cell layer on the petri dish surfaces was approximately $8 \mu \mathrm{m}$, corresponding to a monolayer, 
whereas the integration depth of cells grown on the Matrigel was 20-40 $\mu \mathrm{m}$ after four days of growth. Thus, cells cultured in this 3D environment represent better the in vivo cellular behavior compared to cells cultured as a monolayer. Cells well-adapted to 3D growth are crucial for successful neuronal transplantation, as shown in several animal studies $[16,17]$. Neurons pregrown as a monolayer or immediately injected as a cell suspension (standard procedure) do not seem adapt to 3D cell growth fast enough in the host tissue, i.e. within the critical period of four days after transplantation reported for e.g. dopamine neurons [18]. Merely 5-20\% of the neurons survive after grafting [19] as a result of lack or loss of extracellular matrix attachment, which immediately triggers apoptosis [20]. The development of a 3D scaffold for neuronal cell growth and support in the crucial initial host integration phase could bring significant improvements in survival rate, necessary for neuronal transplantation to become a meaningful therapy.

\subsection{Imaging of neurites}

In order to form an understanding for how neurons interact with different scaffold materials, it is important to be able to visualize these mechanisms not only at cellular level but also for the neurites. Hence, it is encouraging to note the great detail with which neurites protruding from the cell bodies can be visualized by CARS microscopy (Figs. 4A and 4C). Fine features in neurite morphology of sub-micron size can be resolved, as seen in the corresponding close-up images in Figs. 4B and D, where neurite growth cones and even dendritic spines are visible. Dendritic spines are sites of information processing and storage and where most excitatory synapses in the mammalian brain are formed [21], and the ability to visualize such functionally important subunits, see Fig. 4D (arrow), opens up for interesting future studies. The growth cone has a broad sheet-like extension containing protrusions with which the distal end of the neurite adheres to a substrate and explores the surrounding environment [22]. This is a process that has mainly been studied in monolayer cultures on 2D surface $[23,24]$. In order to gain deeper insight into how the mechanisms are in vivo, it is important to study these phenomena in a system that closer resembles the native environment of the cells such as $3 \mathrm{D}$ culture systems.
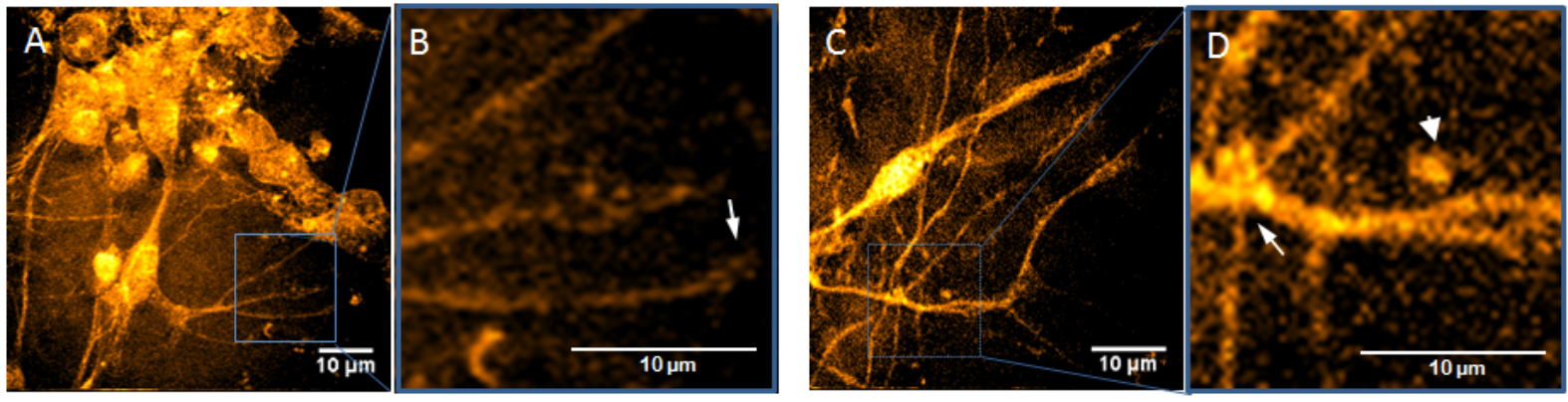

Figure 4. CARS images of neurons and neurites grown for four days on Matrigel. (A) A collection of neurons with neurites protruding from the cell bodies. Neurites highlighted in the square in the lower part of the image, are shown in the close-up image in (B). The growth cone of a neurite (arrow) can here be distinguished. More peripheral neurites with extensive branching can be observed in (C). A close up of the neurites in the squared area is displayed in (D), showing a dendritic spine (arrowhead) and a synapse (arrow). Images are an overlay of several images from a Z-stack. 


\subsection{Neurite growth in 2D and 3D surface}

In order to investigate differences in neurite growth patterns on a $2 \mathrm{D}$ surface and within a $3 \mathrm{D}$ matrix, the neurites of rat primary neurons grown in 2D and 3D were traced in CARS volume images, the result of which is shown in Fig. 5.

Neurites from cells grown on 2D surfaces can merely be observed in the same plane as the cell body, spanning a limited depth of $9 \mu \mathrm{m}$, as shown in the volume rendering in Fig. 5A. The depth color coded image in Fig. 5B displays all neurites in magenta, corresponding to the plane immediately at the surface of the petri dish. Thus, the neurites seem to attach to and align along the supporting surface. The architecture of neurites of cells growing in Matrigel, is significantly different as exemplified in the volume renderings in Figs. 5D-E (different viewpoints). An extensive 3D network of neurites can be observed with longer and more branched neurites, allowing for better connectivity. The average length of the neurites in the Matrigel is estimated to $34 \mu \mathrm{m}$ and the longest traced neurite is $80 \mu \mathrm{m}$ in length. It can be concluded that not only the cells but also the neurites integrate deep into the matrix. This is further confirmed by the depth color coded image in Fig. 5C, displaying several neurites in magenta corresponding to a depth of $42 \mu \mathrm{m}$.
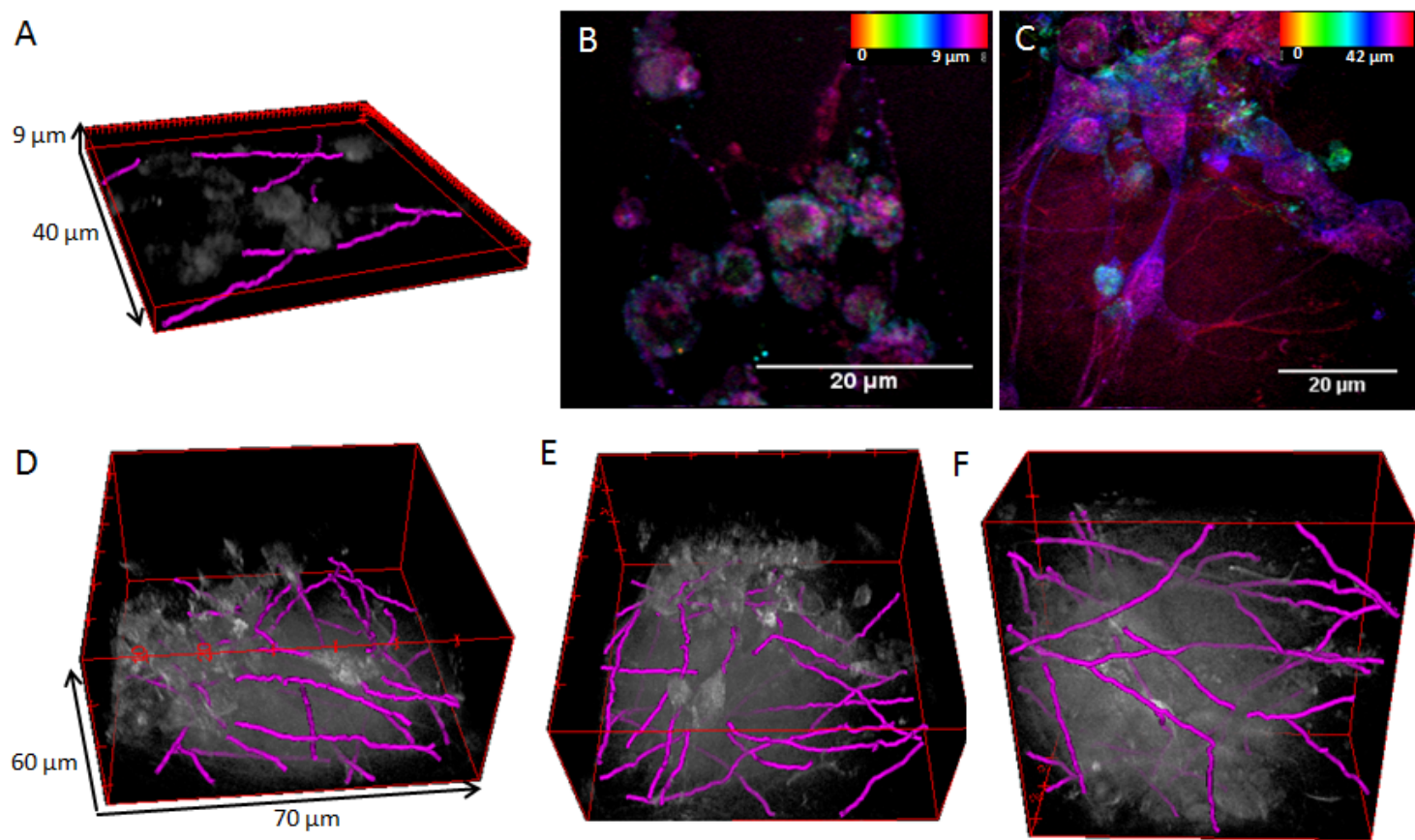

Figure 5. Neurite traces (highlighted in magenta) extracted from CARS microscopy images of rat primary neurons grown on a 2D surface and a 3D hydrogel (Matrigel), respectively. (A-B) Cells grown on a 2D surface and (C-F) a 3D Matrigel matrix. Volume views (A, D-F) are reconstructed from z-stacks of CARS microscopy images. (D-F) show neurite traces in a Matrigel scaffold viewed from different angles: side view, from below and from above. (B) and (C) show depth color coded images from z-stack of cells grown on a 2D surface and the Matrigel, respectively, where the different colors represent different depths within the image.

\section{CONCLUSIONS}

This work suggests that non-linear microscopy has a strong potential to serve as an important instrument in the investigation of neuronal cell growth and development in biopolymeric 
scaffolds for the evaluation of the cell interaction mechanisms with the scaffold materials and the development of novel scaffold materials optimized for the establishment of 3D neural networks and implants.

\section{ACKNOWLEDGEMENTS}

The financial support from the Swedish Research Council is gratefully acknowledged.

\section{REFERENCES}

[1] E. Cukierman, R. Pankov, D. R. Stevens et al., "Taking cell-matrix adhesions to the third dimension," Science, 294(5547), 1708-1712 (2001).

[2] J. J. Pancrazio, J. P. Whelan, D. A. Borkholder et al., "Development and application of cell-based biosensors," Annals of Biomedical Engineering, 27(6), 697-711 (1999).

[3] A. Desai, W. S. Kisaalita, C. Keith et al., "Human neuroblastoma (SH-SY5Y) cell culture and differentiation in 3-D collagen hydrogels for cell-based biosensing," Biosensors \& Bioelectronics, 21(8), 1483-1492 (2006).

[4] T. Xu, C. A. Gregory, P. Molnar et al., "Viability and electrophysiology of neural cell structures generated by the inkjet printing method," Biomaterials, 27(19), 3580-3588 (2006).

[5] H. W. T. Matthew, [Polymers for tissue engineering scaffolds] Marcel Dekker, Inc, New York, 8 (2002).

[6] B. Geiger, A. Bershadsky, R. Pankov et al., "Transmembrane extracellular matrix-cytoskeleton crosstalk," Nature Reviews Molecular Cell Biology, 2(11), 793-805 (2001).

[7] C. Selhuber-Unkel, T. Erdmann, M. Lopez-Garcia et al., "Cell adhesion strength is controlled by intermolecular spacing of adhesion receptors," Biophysical Journal, 98, 543-551 (2010).

[8] R. M. Abeysekera, A. W. Robards, A. B. Hodgson et al., "Improved visualization of folded collagen alpha-chains by ultra-rapid freezing," International Journal of Biological Macromolecules, 15(5), 313-315 (1993).

[9] Z. F. Shao, J. Mou, D. M. Czajkowsky et al., "Biological atomic force microscopy: What is achieved and what is needed," Advances in Physics, 45(1), 1-86 (1996).

[10] J. R. Colvin, and D. E. Witter, "Congo Red and Calcofluor White inhibition of Acetobacter Xylinum cell-growth and of bacterial cellulose microfibril formation - isolation and properties of a transient, extracellular glucan related to cellulose," Protoplasma, 116(1), 24-40 (1983).

[11] R. O. Calderon, B. Attema, and G. H. Devries, "Lipid composition of neuronal cell-bodies and neurites from cultured dorsal-root ganglia," Journal of Neurochemistry, 64(1), 424-429 (1995).

[12] A. Enejder, C. Brackmann, and F. Svedberg, "Coherent Anti-Stokes Raman Scattering Microscopy of Cellular Lipid Storage," Ieee Journal of Selected Topics in Quantum Electronics, 16(3), 506-515 (2010).

[13] C. Krafft, L. Neudert, T. Simat et al., "Near infrared Raman spectra of human brain lipids," Spectrochimica Acta Part a-Molecular and Biomolecular Spectroscopy, 61(7), 1529-1535 (2005).

[14] R. C. Spiker, and I. W. Levin, "Raman spectra and vibrational assignments for dipalmitoyl phosphatidylcholine and structurally related molecules " Biochimica Et Biophysica Acta, 388(3), 361-373 (1975).

[15] G. E. Walrafen, "Raman spectral studies of water structure " Journal of Chemical Physics, 40(11), 3249-3256 (1964).

[16] E. D. Clarkson, W. M. Zawada, F. S. Adams et al., "Strands of embryonic mesencephalic tissue show greater dopamine neuron survival and better behavioral improvement than cell suspensions after transplantation in parkinsonian rats," Brain Research, 806(1), 60-68 (1998). 
[17] M. Emgard, U. Hallin, J. Karlsson et al., "Both apoptosis and necrosis occur early after intracerebral grafting of ventral mesencephalic tissue: a role for protease activation," Journal of Neurochemistry, 86(5), 1223-1232 (2003).

[18] C. E. Sortwell, M. R. Pitzer, and T. J. Collier, "Time course of apoptotic cell death within mesencephalic cell suspension grafts: Implications for improving grafted dopamine neuron survival," Experimental Neurology, 165(2), 268-277 (2000).

[19] P. Brundin, and A. Bjorklund, "Survival, growth and function of dopaminergic neurons grafted to the brain," Progress in Brain Research D, 71, 293-308 (1987).

[20] F. T. Thomas, J. L. Contreras, G. Bilbao et al., "Anoikis, extracellular matrix, and apoptosis factors in isolated cell transplantation," Surgery, 126(2), 299-304 (1999).

[21] P. Hotulainen, and C. C. Hoogenraad, "Actin in dendritic spines: connecting dynamics to function," Journal of Cell Biology, 189(4), 619-629 (2010).

[22] S.-M. Kim, J. Bae, I. H. Cho et al., "Control of growth cone motility and neurite outgrowth by SPIN90," Experimental Cell Research, 317(16), 2276-2287 (2011).

[23] A. C. Greene, C. M. Washburn, G. D. Bachand et al., "Combined chemical and topographical guidance cues for directing cytoarchitectural polarization in primary neurons," Biomaterials, 32(34), 8860-8869 (2011).

[24] C. G. Dotti, C. A. Sullivan, and G. A. Banker, "The establishment of polarity by hippocampalneurons in culture," Journal of Neuroscience, 8(4), 1454-1468 (1988). 